\title{
Xanthine oxidase inhibitory activity of a new isocoumarin obtained from Marantodes pumilum var. pumila leaves
}

Nor-Ashila Aladdin ${ }^{1 \dagger}$, Khairana Husain $^{1 \dagger}$, Juriyati Jalil ${ }^{1}$, Carla Wulandari Sabandar ${ }^{2}$ and Jamia Azdina Jamal ${ }^{1{ }^{*+}}$ (D)

\begin{abstract}
Background: In traditional Malay medicine, Marantodes pumilum (Blume) Kuntze (family Primulaceae) is commonly used by women to treat parturition, flatulence, dysentery, dysmenorrhea, gonorrhea, and bone diseases. Preliminary screening of some Primulaceae species showed that they possess xanthine oxidase inhibitory activity. Thus, this study aimed to investigate the xanthine oxidase inhibitory activity of three varieties of M. pumilum and their phytochemical compounds.
\end{abstract}

Method: Dichloromethane, methanol, and water extracts of the leaves and roots of M. pumilum var. alata, M. pumilum var. pumila, and M. pumilum var. lanceolata were tested using an in vitro xanthine oxidase inhibitory assay. Bioassay-guided fractionation and isolation were carried out on the most active extract using chromatographic techniques. The structures of the isolated compounds were determined using spectroscopic techniques.

Results: The most active dichloromethane extract of M. pumilum var. pumila leaves $\left(I C_{50}=161.6 \mu \mathrm{g} / \mathrm{mL}\right)$ yielded one new compound, 3,7-dihydroxy-5-methoxy-4,8-dimethyl-isocoumarin (1), and five known compounds, viz. ardisiaquinone A (2), maesanin (3), stigmasterol (4), tetracosane (5), and margaric acid (6). The new compound was found to be the most active xanthine oxidase inhibitor with an $I_{50}$ value of $0.66 \pm 0.01 \mu \mathrm{g} / \mathrm{mL}$, which was not significantly different $(p>0.05)$ from that of the positive control, allopurinol $\left(I C_{50}=0.24 \pm 0.00 \mu \mathrm{g} / \mathrm{mL}\right)$.

Conclusion: This study suggests that the new compound 3,7-dihydroxy-5-methoxy-4,8-dimethyl-isocoumarin (1), which was isolated from the dichloromethane extract of $M$. pumilum var. pumila leaves, could be a potential xanthine oxidase inhibitor.

Keywords: Marantodes pumilum var. pumila, Xanthine oxidase inhibitor, Isocoumarin, Hyperuricemia, Gout

\footnotetext{
* Correspondence: jamia@ukm.edu.my

${ }^{\dagger}$ Nor-Ashila Aladdin, Khairana Husain and Jamia Azdina Jamal contributed equally to this work.

${ }^{1}$ Drug and Herbal Research Centre, Faculty of Pharmacy, Universiti Kebangsaan Malaysia, Kuala Lumpur, Malaysia

Full list of author information is available at the end of the article
}

(c) The Author(s). 2020 Open Access This article is licensed under a Creative Commons Attribution 4.0 International License, which permits use, sharing, adaptation, distribution and reproduction in any medium or format, as long as you give appropriate credit to the original author(s) and the source, provide a link to the Creative Commons licence, and indicate if changes were made. The images or other third party material in this article are included in the article's Creative Commons licence, unless indicated otherwise in a credit line to the material. If material is not included in the article's Creative Commons licence and your intended use is not permitted by statutory regulation or exceeds the permitted use, you will need to obtain permission directly from the copyright holder. To view a copy of this licence, visit http://creativecommons.org/licenses/by/4.0/. The Creative Commons Public Domain Dedication waiver (http://creativecommons.org/publicdomain/zero/1.0/) applies to the data made available in this article, unless otherwise stated in a credit line to the data. 


\section{Background}

Marantodes pumilum (Blume) Kuntze belongs to the Primulaceae family [1]. It was previously known as Labisia pumila (Blume) Fern.-Vill. from the Myrsinaceae family [2]. The taxonomic characteristics of eight varieties of M. pumilum have been described [3], and three of the varieties (var. alata Scheff., var. pumila, and var. lanceolata (Scheff.) Mez) are commonly used in Malaysia [2]. The close resemblance of var. alata and var. pumila leaves has made macromorphological identification quite difficult, as the leaf laminas of both varieties are either narrowly or broadly elliptic or ovate with $10-30 \times 1.3-11 \mathrm{~cm}$ dimensions [3]. However, their petioles differ. The petiole of var. alata is $5-12 \mathrm{~cm}$ long and broadly winged (3-5 mm wide), whereas that of var. pumila is $4-15 \mathrm{~cm}$ long and slightly winged. Nonetheless, to differentiate them based on characteristic anatomical features and chemical profiling, a pharmacognostical study of these varieties was performed using microscopic, highperformance thin layer chromatography (HPTLC), high performance liquid chromatography (HPLC), and attenuated total reflectance-Fourier transform infrared spectroscopy (ATR-FTIR) techniques [4].

In traditional Malay medicine, $M$. pumilum decoction is popularly used among women to induce and facilitate labor, delay fertility, and regain vitality, as well as to treat flatulence, dysentery, dysmenorrhea, gonorrhea, and bone diseases [5-7]. Men of several ethnic groups in the Sarawak state of Malaysia also consume the plant to maintain and increase stamina [8]. Additionally, the plant has been increasingly used as a supplement and beverage among the public for general health maintenance [9]. Previous scientific studies have reported the activities of M. pumilum, including antioxidant [10], xanthine oxidase inhibition [11, 12], antimicrobial [13], anti-inflammatory [14], uterotonic effect [15], phytoestrogenicity [16], anti-obesity [17], anti-aging [18], and anti-carcinogenic [19]. Its phytochemical compositions such as triterpenoid saponins, alk(en) ylresorcinols, benzoquinone derivatives, fatty acids, flavonoids, and phenolics, have been documented [13, 20-23].

Xanthine oxidase (XO) catalyzes the oxidation of hypoxanthine to xanthine and xanthine to uric acid [24]. It plays a major role during the last step of purine nucleotide metabolism in humans, and serves as an important biological source of oxygen-derived free radicals. Free radicals can contribute to the oxidative damage to living tissues, which are involved in many pathological processes and various ischemic tissues, vascular injuries, and inflammation $[25,26]$. Xanthine oxidase is primarily distributed in the liver and intestine [27]. In humans, overproduction of xanthine oxidase elevates the blood stream uric acid concentration and leads to hyperuricemia [28]. Uric acid deposition begins when uric acid dissolves in the blood and forms urate monohydrate crystals in the joints and kidneys, leading to painful inflammation. Uric acid has been identified as a marker for gout and several metabolic and hemodynamic abnormalities $[25,29,30]$. Synthetic xanthine oxidase inhibitors such as allopurinol, febuxostat, and phenylpyrazol derivative Y-700, have been widely used to treat hyperuricemia and gout [27], but may have side effects. The extensively prescribed allopurinol has been reported to cause Stevens-Johnson syndrome, toxic epidermal necrolysis, hepatic disorders, and renal dysfunction [31]. Therefore, new alternatives such as medicinal plants, with fewer side effects, are desired [32, 33].

Phytochemical constituents such as phenolics, flavonoids, coumarins, lignans, triterpenoids, and alkaloids have been reported to inhibit xanthine oxidase [27, 34-36]. Esculetin, a hydroxycoumarin derivative, displayed strong xanthine oxidase inhibitory activity [37] and was proposed as an appropriate bioactive quality control marker for a traditional Chinese medicine formula used in the treatment of hyperuricemia [38]. The extract of M. pumilum was reported to alleviate hyperuricemia in vivo [39]. Thus, in this study, potential xanthine oxidase inhibitors were determined by evaluating the xanthine oxidase inhibitory activity of $M$. pumilum varieties and isolated compounds using an in vitro assay. The compound could be used as an analytical marker for quality control purposes of M. pumilum-containing herbal products intended for hyperuricemia or gouty conditions.

\section{Methods}

\section{Materials and equipment}

Microplates (96-well) used in the in vitro assay were obtained from Thermo Multiskan Go (Waltham, MA, USA). The following adsorbents were used: silica gel 60 (5-40 $\mu \mathrm{m}$, cat. no. 1.07747) was used for vacuum liquid chromatography (VLC), silica gel $60(40-63 \mu \mathrm{m}$, cat. no. 1.09385) and Sephadex LH-20 (GE Healthcare, Upsalla, Sweden) were used for column chromatography (CC), and silica gel 60 GF254 (0.25 mm, cat. no. 1.05554) was used for thin layer chromatography (TLC). The silica gels were obtained from Merck (Darmstadt, Germany).

For structural elucidation of the isolated compounds, ultraviolet (UV) spectra were recorded in ethanol using a Shimadzu UV1800 UV-Vis spectrophotometer (Shimadzu Corp., Kyoto, Japan), and infra-red (IR) spectra were obtained using a Spectrum 100 FTIR spectrophotometer (PerkinElmer, Inc., Waltham, MA, USA) with an ATR technique. One-dimensional proton $\left({ }^{1} \mathrm{H}\right)$ and carbon $\left({ }^{13} \mathrm{C}\right)$ and two-dimensional nuclear magnetic resonance (NMR) spectra were determined using a Bruker Avance III $600 \mathrm{MHz}$ spectrometer (Bruker BioSpin, Karlsruhe, Germany), while high-resolution electrospray ionization mass spectrometry (HR-ESI-MS) and electron 
ionization mass spectrometry (EI-MS) spectra were obtained using an Ultimate 3000 system, MicrOTOF-Q II (Bruker Daltonics, Bremen, Germany).

\section{Chemicals and reagents}

Analytical grade organic solvents, including dichloromethane $(\mathrm{DCM})$, methanol $(\mathrm{MeOH})$, chloroform $\left(\mathrm{CHCl}_{3}\right)$, dimethyl sulfoxide (DMSO), hexane, ethyl acetate (EtOAc), toluene, acetone, and ethanol (EtOH), were purchased from Merck (Darmstadt, Germany). For the bioassay, allopurinol, xanthine, and xanthine oxidase (cow's milk) were purchased from Sigma-Aldrich Chemicals (St. Louis, MO, USA), while dimethyl sulfoxide (DMSO), hydrochloric acid $(\mathrm{HCl})$, sodium hydroxide $(\mathrm{NaOH})$, and potassium dihydrogen phosphate $\left(\mathrm{KH}_{2} \mathrm{PO}_{4}\right)$ were purchased from Merck (Darmstadt, Germany).

\section{Preparation of $M$. pumilum extracts}

Three wild varieties of $M$. pumilum were collected from the Bujang Melaka Forest Reserve in Malaysia and authenticated by Mr. Sani Miran ${ }^{\dagger}$, a botanist from the Herbarium of Universiti Kebangsaan Malaysia in Bangi (UKMB). The voucher specimens of var. alata (voucher number: UKMB 30006/SM 2622), var. pumila (UKMB 30007/SM s.n.), and var. lanceolata (UKMB 30008/SM s.n.) were deposited in the Herbarium of Universiti Kebangsaan Malaysia.

Leaves and roots (consisting of both stems and roots) of the fresh plants were separated and air-dried under shade. Following this, they were coarsely ground to obtain six powdered plant materials: var. alata leaves $(0.2$ $\mathrm{kg})$ and roots $(0.8 \mathrm{~kg})$, var. pumila leaves $(0.8 \mathrm{~kg})$ and roots $(2.0 \mathrm{~kg})$, and var. lanceolata leaves $(0.2 \mathrm{~kg})$ and roots $(0.5 \mathrm{~kg})$. Within 1 week, each plant powder was successively macerated with dichloromethane in a powder-to-solvent ratio of 1:5, followed by methanol (ratio of 1:5). The methanol residue was refluxed with distilled water in a residue-to-solvent ratio of 1:13 for the leaves and 1:10 for the roots. The dichloromethane and methanol fluid extracts were vacuum-dried, and the water extracts were freeze-dried. This process resulted in eighteen dried extracts, which were stored in a refrigerator at $4{ }^{\circ} \mathrm{C}$ until further analyses.

\section{In vitro xanthine oxidase assay}

The xanthine oxidase inhibitory assay was carried out using a previously reported method [40] with slight modifications. Initially, allopurinol (the positive control) and the dichloromethane and methanol extracts were dissolved in dimethyl sulfoxide (DMSO), and the water extracts were dissolved in distilled water. This was followed by dilution with potassium phosphate buffer $(0.05 \mathrm{M}, \mathrm{pH} 7.5)$ to achieve the desired concentrations. Each test solution contained $0.5 \%$ DMSO. The assay was performed in triplicates in a 96-well microplate. The assay reaction mixture, which consisted of $130 \mu \mathrm{L}$ of buffer, $10 \mu \mathrm{L}$ of either test solution $(400 \mu \mathrm{g} / \mathrm{mL}$ for extracts and $100 \mu \mathrm{g} / \mathrm{mL}$ for isolated compounds) or allopurinol $(100 \mu \mathrm{g} / \mathrm{mL})$, and $10 \mu \mathrm{L}$ of xanthine oxidase $(0.2 \mathrm{U} /$ well $)$, was incubated at $25^{\circ} \mathrm{C}$ for $15 \mathrm{~min}$. Then, $100 \mu \mathrm{L}$ of substrate solution, xanthine $(0.15 \mathrm{mM}, \mathrm{pH} 7.5)$, was added before further incubating at $25^{\circ} \mathrm{C}$ for $10 \mathrm{~min}$. The final assay mixture was spectrophotometrically measured at $295 \mathrm{~nm}$. Xanthine oxidase inhibitory activity was expressed as the percentage of xanthine oxidase inhibition and calculated using the following formula:

$$
\begin{aligned}
& \text { \%Xanthine Oxidase Inhibition } \\
& \quad=\left[\frac{(A-B)-(C-D)}{(A-B)}\right] \times 100
\end{aligned}
$$

Where A is the optical density without the test solution or allopurinol, B is the optical density of blank solution containing only potassium phosphate buffer $(0.05$ $\mathrm{M}, \mathrm{pH} 7.5), \mathrm{C}$ is the optical density of the test solution or allopurinol with the presence of xanthine oxidase, and $\mathrm{D}$ is the optical density of the test solution or allopurinol without xanthine oxidase. Test solutions with more than $50 \%$ xanthine oxidase inhibition were reassayed at concentrations of $25,50,100,200$, and $400 \mu \mathrm{g} /$ $\mathrm{mL}$ for extracts, $0.39,0.78,1.56,3.13,6.25,12.5,25,50$ and $100 \mu \mathrm{g} / \mathrm{mL}$ for compound $\mathbf{1}$, and $6.25,12.5,25,50$, and $100 \mu \mathrm{g} / \mathrm{mL}$ for compound 2 . Their half-maximal inhibitory concentration $\left(\mathrm{IC}_{50}\right)$ values were determined from percentages of xanthine oxidase inhibition of the respective concentration range using GraphPad Prism 5 software (La Jolla, CA, USA) and compared with that of allopurinol (0.0064, 0.032, 0.16, 0.8, 4, 20, and $100 \mu \mathrm{g} /$ $\mathrm{mL})$.

\section{Isolation and structural elucidation of compounds from M. pumilum var. pumila}

The screening assay revealed that the dichloromethane extract of M. pumilum var. pumila leaves was most active. The extract $(20.0 \mathrm{~g})$ was fractionated by vacuum liquid chromatography using silica gel and gradient elution with increasing polarity mobile phase, that is, $3 \mathrm{~L}$ of hexane-ethyl acetate $(9: 1,8: 2,7: 3,6: 4,5: 5,3: 7,2: 8$, and 1:9) followed by $2 \mathrm{~L}$ of $100 \%$ ethyl acetate and $2 \mathrm{~L}$ of $100 \%$ methanol. Eluents $(250 \mathrm{~mL}$ each) were collected and combined based on the similarity of TLC profiles to obtain 16 fractions $\left(\mathrm{CC} 1: \mathrm{F}_{1-16}\right)$ (Fig. 1). The fractions were further fractionated using various chromatographic techniques with different solvent compositions to obtain six pure compounds. 


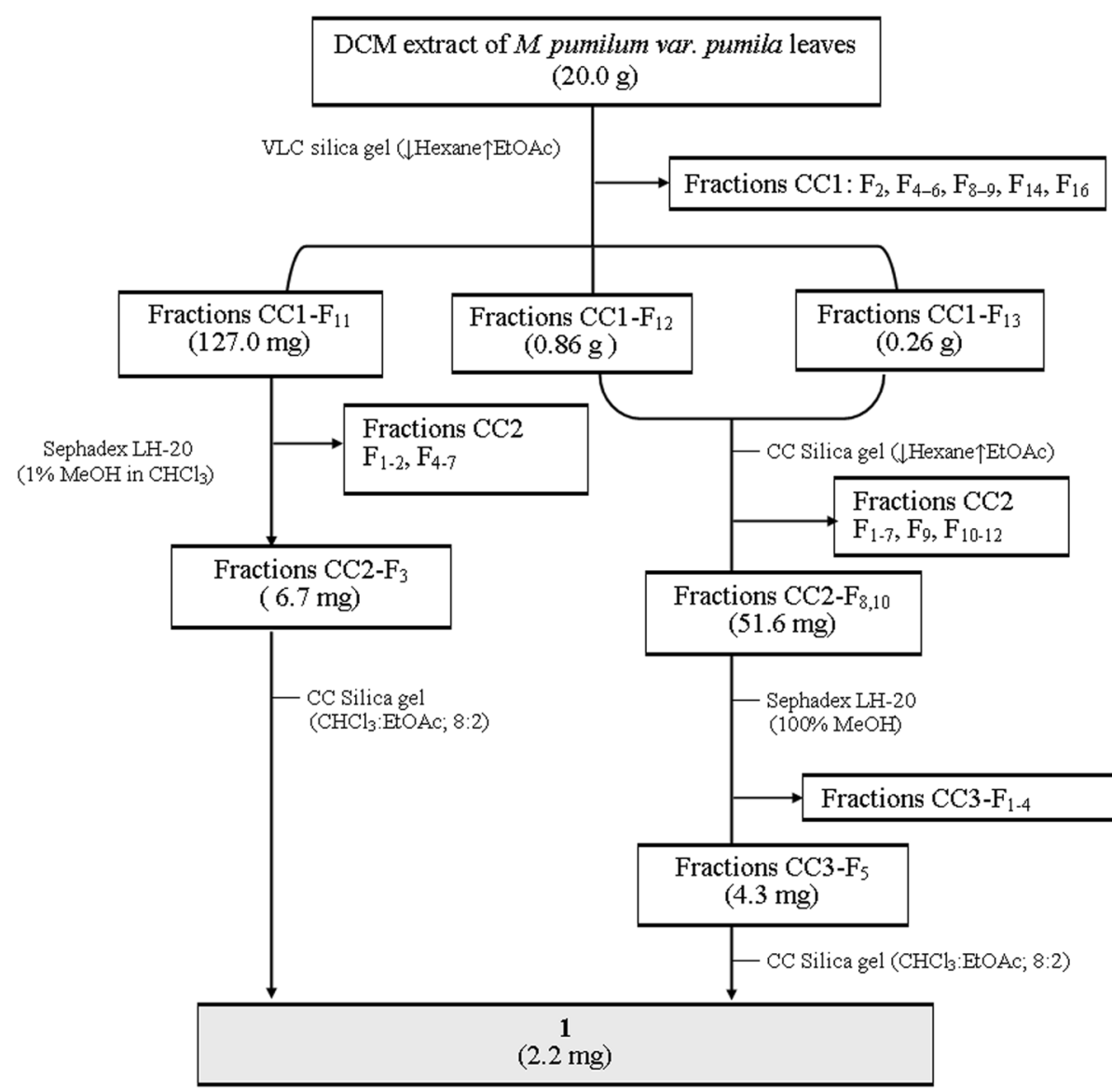

Fig. 1 Flowchart of bioassay-guided isolation of compound 1 from dichloromethane extract of Marantodes pumilum var. pumila leaves

\section{3,7-dihydroxy-5-methoxy-4,8-dimethyl-isocoumarin (1)}

The fraction $\mathrm{CC} 1-\mathrm{F}_{11}(0.13 \mathrm{~g})$ was fractionated using Sephadex LH-20 column chromatography (Ф $25 \mathrm{~mm})$ with $1 \%$ methanol in chloroform to yield seven fractions that were assayed for xanthine oxidase inhibition. The third fraction was then eluted using silica gel column chromatography with chloroform-ethyl acetate (4:1) to obtain pure compound 1. Compound $\mathbf{1}$ was also isolated from fractions $\mathrm{CC} 1-\mathrm{F}_{12}(0.86 \mathrm{mg})$ and $\mathrm{CC} 1-\mathrm{F}_{13}(0.26 \mathrm{mg})$ via several steps of bioassay-guided column chromatography and xanthine oxidase inhibition assay (Fig. 1). Compound $\mathbf{1}$ was obtained as a white amorphous solid $(2.2 \mathrm{mg})$, and the data for its structural elucidation were as follows: TLC: $R_{\mathrm{f}} 0.3$ (tolueneacetone, 9:1); UV (EtOH) $\lambda_{\max } \mathrm{nm}(\log \varepsilon): 275$ (3.17); IR (ATR) ${ }_{\max }, \mathrm{cm}^{-1}: 3256,2926,2952,1734,1659,1607$, 1464, 1383, 1311, and 1202; EI-MS $m / z: 236\left[\mathrm{M}^{+}\right.$(calculated for $\left.\mathrm{C}_{12} \mathrm{H}_{12} \mathrm{O}_{5}, 236.2207 \mathrm{~g} / \mathrm{mol}\right)$; ${ }^{1} \mathrm{H}-\mathrm{NMR}\left(\mathrm{CDCl}_{3}\right.$, $600 \mathrm{MHz}) \delta_{\mathrm{H}}(\mathrm{ppm}): 2.45(3 \mathrm{H}, s, \mathrm{H}-11), 2.65(3 \mathrm{H}, s, \mathrm{H}-$ 12), $3.89\left(s, \mathrm{OCH}_{3}\right)$, and $6.91(s, \mathrm{H}-6) ;{ }^{13} \mathrm{C}-\mathrm{NMR}\left(\mathrm{CDCl}_{3}\right.$, $150 \mathrm{MHz}) \delta_{\mathrm{C}}$ (ppm): 162.7 (C-1), 157.1 (C-3), 139.9 (C-4), 110.2 (C-4a), 153.1 (C-5), 101.8 (C-6), 142.5 (C-7), 119.8 (C-8), 131.1 (C-8a), 18.2 (C-9), and 15.2 (C-10).

\section{Ardisiaquinone A (2)}

The fraction $\mathrm{CC} 1-\mathrm{F}_{15}(0.69 \mathrm{~g})$ was fractionated using Sephadex LH-20 column chromatography with $1 \%$ methanol in chloroform to yield eight fractions. The third fraction was then eluted using silica gel column chromatography with chloroform-methanol (9:1) to obtain five fractions. The fourth fraction was triturated with hexane-methanol (1:1) to give compound 2. Compound $\mathbf{2}$ was obtained as a yellow powder $(5.0 \mathrm{mg})$, and the data for its structural elucidation were as follows: UV (EtOH) $\lambda_{\max } \mathrm{nm}(\log \varepsilon): 285$ (3.15), and 206 (3.00); IR (ATR) $\max \mathrm{cm}^{-1}: 3346,3342,2923,2954,1633,1595$, 1463, 1311, 1202, 1078, and 838; HR-ESI-MS (+ve mode) $\mathrm{m} /$ $z: 527.4275[\mathrm{M}-\mathrm{H}]^{+}$(calculated for $\mathrm{C}_{30} \mathrm{H}_{40} \mathrm{O}_{8}, 528.4275 \mathrm{~g} /$ mol); ${ }^{1} \mathrm{H}-\mathrm{NMR}\left(\mathrm{CDCl}_{3}, 600 \mathrm{MHz}\right) \delta_{\mathrm{H}}(\mathrm{ppm}): 1.29(16 \mathrm{H}, \mathrm{m}$, H-9-H-12, H-9'-H-12', overlapped), 1.47 (4H, $\left.m, \mathrm{H}-8, \mathrm{H}-8^{\prime}\right)$, $2.02\left(4 \mathrm{H}, m, \mathrm{H}-13, \mathrm{H}-13^{\prime}\right), 2.46\left(4 \mathrm{H}, m, \mathrm{H}-7, \mathrm{H}-7^{\prime}\right), 3.88$ (s, $\left.\mathrm{OCH}_{3}\right), 5.35\left(2 \mathrm{H}, m, \mathrm{H}-14, \mathrm{H}-14^{\prime}\right), 5.86(d, J=3.0 \mathrm{~Hz}, \mathrm{H}-6$, $\left.\mathrm{H}-6^{\prime}\right)$, and $7.28\left(\right.$ br s, OH); ${ }^{13} \mathrm{C}-\mathrm{NMR}\left(\mathrm{CDCl}_{3}, 150 \mathrm{MHz}\right) \delta_{\mathrm{C}}$ (ppm): 22.6 (C-7, C-7'), 27.2 (C-13, C-13'), 27.9 (C-8, C-8'), 29.2-29.7 (C-9-12, C-9'-12'), 129.7 (C-14, C-14'), 182.8 (C-1, C-1'), 151.5 (C-2, C-2'), 119.1 (C-3, C-3'), 181.7 (C-4, C-4'), 161.1 (C-5, C-5'), and 102.2 (C-6, C-6'). 
Table 1 Percentages of xanthine oxidase inhibition of extracts of Marantodes pumilum varieties and allopurinol

\begin{tabular}{|c|c|c|c|c|}
\hline Species & Plant part & Crude extracts & Yield (\%) & Percentage of xanthine oxidase inhibition (\%) ${ }^{a}$ \\
\hline \multirow[t]{6}{*}{ M. pumilum var. alata } & Roots & DCM & 0.85 & 0.00 \\
\hline & & $\mathrm{MeOH}$ & 8.08 & 0.00 \\
\hline & & $\mathrm{H}_{2} \mathrm{O}$ & 6.00 & 0.00 \\
\hline & Leaves & DCM & 1.80 & $68.21 \pm 2.50$ \\
\hline & & $\mathrm{MeOH}$ & 2.23 & $41.15 \pm 4.31$ \\
\hline & & $\mathrm{H}_{2} \mathrm{O}$ & 2.74 & 0.00 \\
\hline \multirow[t]{6}{*}{ M. pumilum var. pumila } & Roots & DCM & 1.59 & 0.00 \\
\hline & & $\mathrm{MeOH}$ & 4.63 & 0.00 \\
\hline & & $\mathrm{H}_{2} \mathrm{O}$ & 2.58 & 0.00 \\
\hline & Leaves & DCM & 1.38 & $85.77 \pm 0.70$ \\
\hline & & $\mathrm{MeOH}$ & 1.09 & $80.97 \pm 0.72$ \\
\hline & & $\mathrm{H}_{2} \mathrm{O}$ & 4.56 & 0.00 \\
\hline \multirow[t]{6}{*}{ M. pumilum var. lanceolata } & Roots & DCM & 3.16 & 0.00 \\
\hline & & $\mathrm{MeOH}$ & 7.21 & 0.00 \\
\hline & & $\mathrm{H}_{2} \mathrm{O}$ & 5.16 & 0.00 \\
\hline & Leaves & DCM & 3.30 & $74.33 \pm 4.33$ \\
\hline & & $\mathrm{MeOH}$ & 8.70 & $67.52 \pm 0.35$ \\
\hline & & $\mathrm{H}_{2} \mathrm{O}$ & 1.03 & 0.00 \\
\hline Allopurinol (positive control) & & & & $99.82 \pm 0.00$ \\
\hline
\end{tabular}

Data are presented as mean \pm S.E.M. of three replicates $(n=3)$

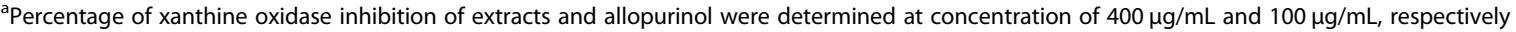

\section{Maesanin (3)}

The fraction $\mathrm{CC} 1-\mathrm{F}_{3}(0.62 \mathrm{~g})$ was fractionated using Sephadex LH-20 column chromatography with $1 \%$ methanol in chloroform to yield eight fractions. The fourth fraction was then eluted using silica gel column chromatography with chloroform-ethyl acetate (9:1), followed by trituration with hexane-methanol (1:1) to obtain compound 3. Compound 3 was obtained as a yellow crystal $(10.0 \mathrm{mg})$, and the data for its structural elucidation were as follows: UV (EtOH) $\lambda_{\max }$ $\mathrm{nm}(\log \varepsilon): 285$ (3.17) and 206 (2.97); IR (ATR) $\max , \mathrm{cm}^{-1}$ : 3342, 2851, 2921, 1659, 1607, 1464, 1383, 1311, and 1200; HR-ESI-MS $m / z: \quad 363.5800 \quad[\mathrm{M}+\mathrm{H}]^{+} \quad$ (calculated for $\mathrm{C}_{22} \mathrm{H}_{34} \mathrm{O}_{4}, 362.5800 \mathrm{~g} / \mathrm{mol}$ ); ${ }^{1} \mathrm{H}-\mathrm{NMR}\left(\mathrm{CD}_{3} \mathrm{OD}, 600 \mathrm{MHz}\right.$ ) $\delta_{\mathrm{H}}$ (ppm): $0.93\left(3 \mathrm{H}, m, \mathrm{H}-15^{\prime}\right), 1.29-1.34\left(16 \mathrm{H}, m, \mathrm{H}-3^{\prime}-\mathrm{H}-\right.$ 8', H-13'-H-14', overlapped), 1.45 (2H, m, H-2'), 2.05 (4H, $\left.m, \mathrm{H}-9^{\prime}, \mathrm{H}-12^{\prime}\right), 2.41\left(2 \mathrm{H}, t, J=7.8, \mathrm{H}-1^{\prime}\right), 3.85\left(\mathrm{~s}, \mathrm{OCH}_{3}\right)$, $5.36\left(2 \mathrm{H}, m, \mathrm{H}-10^{\prime}, \mathrm{H}-11^{\prime}\right)$, and $5.91(s, \mathrm{H}-6) ;{ }^{13} \mathrm{C}-\mathrm{NMR}$ $\left(\mathrm{CD}_{3} \mathrm{OD}, 150 \mathrm{MHz}\right) \delta_{\mathrm{C}}(\mathrm{ppm}): 13.1\left(\mathrm{C}-15^{\prime}\right), 21.9\left(\mathrm{C}-1^{\prime}\right)$, 22.3-31.5 (C-2'-8', C-13'-14'), 26.7 (C-9', C-12'), 129.4 (C10', C-11'), 182.2 (C-1), 154.5 (C-2), 118.7 (C-3), 183.0 (C4), $160.5(\mathrm{C}-5), 55.8\left(\mathrm{OCH}_{3}\right)$, and $102.6(\mathrm{C}-6)$.

\section{Stigmasterol (4)}

The fraction $\mathrm{CC}_{1}-\mathrm{F}_{7}(2.0 \mathrm{~g})$ was fractionated using Sephadex LH-20 column chromatography with $1 \%$ methanol in chloroform to yield ten fractions. The fifth fraction was purified by re-crystallization in methanol to yield compound $\mathbf{4}$. Compound $\mathbf{4}$ was obtained as a white needle crystal $(14.0 \mathrm{mg})$, and the data for its structural elucidation were as follows: $\mathrm{mp} 133-134^{\circ} \mathrm{C}$; UV $(\mathrm{EtOH})$ $\lambda_{\max } \mathrm{nm}(\log \varepsilon): 202$ (3.76); IR (ATR) $\max , \mathrm{cm}^{-1}: 3347$, 2934, 2868, 1464, 1382, 1048, and 968; HR-ESI-MS $m / z$ : $413.2666[\mathrm{M}+\mathrm{H}]^{+}$(calculated for $\mathrm{C}_{29} \mathrm{H}_{48} \mathrm{O}, 412.2470 \mathrm{~g} /$ $\mathrm{mol}) ;{ }^{1} \mathrm{H}-\mathrm{NMR}\left(\mathrm{CDCl}_{3}, 600 \mathrm{MHz}\right) \delta_{\mathrm{H}}(\mathrm{ppm}): 0.72(3 \mathrm{H}, s$, $\mathrm{H}-18), 0.82(3 \mathrm{H}, d, J=6.6 \mathrm{~Hz}, \mathrm{H}-27), 0.83$ (3H, $t, \mathrm{H}-29)$, $0.87(3 \mathrm{H}, d, J=6.0 \mathrm{~Hz}, \mathrm{H}-26), 0.95(\mathrm{H}-9), 1.01(3 \mathrm{H}, s, \mathrm{H}-$ 19), 1.03 (H-14), $1.04(3 \mathrm{H}, d, J=6.6 \mathrm{~Hz}, \mathrm{H}-21), 1.08(\mathrm{H}-$ 15), 1.10 (H-1), 1.13 (H-17), 1.19 (H-12, H-28), 1.28 (H16), 1.43 (H-28), 1.48 (H-8), 1.50 (H-7), 1.52 (H-2, H11), 1.53 (H-25), 1.54 (H-24), 1.58 (H-15), 1.73 (H-16), 1.86 (H-2), 1.88 (H-1), 1.98 (H-7), 2.01 (H-12), 2.07 (H20), $2.26(\mathrm{H}-4), 2.32(\mathrm{H}-4), 3.55(\mathrm{H}-3), 5.04$ (dd, $J=15.3$, $8.9 \mathrm{~Hz}, \mathrm{H}-23), 5.17$ (dd, $J=15.1,8.7 \mathrm{~Hz}, \mathrm{H}-22)$, and 5.37 $(\mathrm{H}-6) ;{ }^{13} \mathrm{C}-\mathrm{NMR}\left(\mathrm{CDCl}_{3}, 150 \mathrm{MHz}\right) \delta_{\mathrm{C}}(\mathrm{ppm}): 11.9(\mathrm{C}-$ 18), 12.3 (C-29), 19.0 (C-27), 19.4 (C-19), 21.1 (C-11, C26), 23.1 (C-21), 24.3 (C-15), 26.0 (C-28), 29.1 (C-16), 31.7 (C-7, C-25), 31.9 (C-2, C-8), 36.2 (C-10), 37.3 (C-1), 39.7 (C-12), 40.6 (C-20), 42.3 (C-13), 45.8 (C-4), 50.1 (C9), 51.3 (C-24), 56.0 (C-17), 56.8 (C-14), 71.8 (C-3), 121.8 (C-6), 129.3 (C-23), 138.4 (C-22), and 140.8 (C-5).

\section{Tetracosane (5)}

The fraction $\mathrm{CC} 1-\mathrm{F}_{1}(0.12 \mathrm{~g})$ was precipitated to obtain compound 5. Compound $\mathbf{5}$ was obtained as a white 
Table $2 I_{50}$ values of xanthine oxidase inhibition of selected extracts of Marantodes pumilum varieties and allopurinol

\begin{tabular}{|c|c|c|c|c|c|}
\hline Species & $\begin{array}{l}\text { Plant } \\
\text { part }\end{array}$ & $\begin{array}{l}\text { Crude } \\
\text { extracts }\end{array}$ & $\begin{array}{l}\text { Concentration } \\
(\mu \mathrm{g} / \mathrm{mL})\end{array}$ & $\begin{array}{l}\text { Percentage of xanthine oxidase } \\
\text { inhibition (\%) }\end{array}$ & $\begin{array}{l}\mathrm{IC}_{50} \text { value of } x \text { anthine oxidase } \\
\text { inhibition }(\mu \mathrm{g} / \mathrm{mL})^{\mathrm{a}}\end{array}$ \\
\hline \multirow{5}{*}{$\begin{array}{l}\text { M. pumilum var. } \\
\text { alata }\end{array}$} & \multirow[t]{5}{*}{ Leaves } & \multirow[t]{5}{*}{ DCM } & 25 & $28.08 \pm 0.18$ & \multirow[t]{5}{*}{$310.9 \pm 8.25$} \\
\hline & & & 50 & $15.94 \pm 0.00$ & \\
\hline & & & 100 & $3.99 \pm 0.00$ & \\
\hline & & & 200 & $20.49 \pm 0.22$ & \\
\hline & & & 400 & $68.21 \pm 2.50$ & \\
\hline \multirow{10}{*}{$\begin{array}{l}\text { M. pumilum var. } \\
\text { pumila }\end{array}$} & \multirow[t]{10}{*}{ Leaves } & \multirow[t]{5}{*}{ DCM } & 25 & $15.34 \pm 0.25$ & \multirow[t]{5}{*}{$161.6 \pm 7.35$} \\
\hline & & & 50 & $14.31 \pm 0.89$ & \\
\hline & & & 100 & $23.85 \pm 0.05$ & \\
\hline & & & 200 & $65.28 \pm 1.81$ & \\
\hline & & & 400 & $85.77 \pm 0.70$ & \\
\hline & & \multirow[t]{5}{*}{$\mathrm{MeOH}$} & 25 & $4.94 \pm 0.29$ & \multirow[t]{5}{*}{$175.1 \pm 0.20$} \\
\hline & & & 50 & $9.86 \pm 1.51$ & \\
\hline & & & 100 & $20.08 \pm 0.05$ & \\
\hline & & & 200 & $60.73 \pm 0.72$ & \\
\hline & & & 400 & $80.97 \pm 0.72$ & \\
\hline \multirow{10}{*}{$\begin{array}{l}\text { M. pumilum var. } \\
\text { lanceolata }\end{array}$} & \multirow[t]{10}{*}{ Leaves } & \multirow[t]{5}{*}{ DCM } & 25 & $26.90 \pm 3.09$ & \multirow[t]{5}{*}{$233.1 \pm 19.85$} \\
\hline & & & 50 & $13.60 \pm 2.93$ & \\
\hline & & & 100 & $28.89 \pm 0.87$ & \\
\hline & & & 200 & $35.59 \pm 1.40$ & \\
\hline & & & 400 & $74.33 \pm 4.33$ & \\
\hline & & \multirow[t]{5}{*}{$\mathrm{MeOH}$} & 25 & $19.15 \pm 0.04$ & \multirow[t]{5}{*}{$185.3 \pm 2.50$} \\
\hline & & & 50 & $11.25 \pm 1.21$ & \\
\hline & & & 100 & $37.98 \pm 0.88$ & \\
\hline & & & 200 & $53.37 \pm 0.37$ & \\
\hline & & & 400 & $67.52 \pm 0.35$ & \\
\hline \multirow{7}{*}{\multicolumn{2}{|c|}{ Allopurinol (positive control) }} & & 0.0064 & $4.12 \pm 0.00$ & \multirow[t]{7}{*}{$0.24 \pm 0.00$} \\
\hline & & & 0.032 & $17.81 \pm 0.00$ & \\
\hline & & & 0.16 & $29.47 \pm 0.00$ & \\
\hline & & & 0.8 & $93.66 \pm 0.00$ & \\
\hline & & & 4 & $98.86 \pm 0.00$ & \\
\hline & & & 20 & $99.01 \pm 0.00$ & \\
\hline & & & 100 & $99.82 \pm 0.00$ & \\
\hline
\end{tabular}

Data are presented as mean \pm SEM. of three replicates $(n=3)$

${ }^{\mathrm{a}} \mathrm{C}_{50}$ values were obtained based on the percentage of xanthine oxidase inhibition of extracts and allopurinol at different concentrations using the Graphpad Prism 5 software (La Jolla, CA, USA)

waxy solid $(10.8 \mathrm{mg})$, and the data for its structural elucidation were as follows: UV (EtOH) $\lambda_{\max } \mathrm{nm}(\log$ $\varepsilon)$ : 202 (1.35); IR (ATR) $\max ^{-1} \mathrm{~cm}^{-1}$ 2915, 2849, 1473, 1463, 1262, 1021, 1096, 802, 729, and 719; HRESI-MS $m / z: 338.3369\left[\mathrm{M}^{+}\right]$(calculated for $\mathrm{C}_{24} \mathrm{H}_{50}$, $338.3913 \mathrm{~g} / \mathrm{mol}) ; \quad{ }^{1} \mathrm{H}-\mathrm{NMR} \quad\left(\mathrm{CDCl}_{3}, \quad 600 \mathrm{MHz}\right) \quad \delta_{\mathrm{H}}$ (ppm): 0.88 (6H, $m, \mathrm{H}-1, \mathrm{H}-24), 1.27(40 \mathrm{H}, m, \mathrm{H}-3-$ $\mathrm{H}-22)$, and $1.32(4 \mathrm{H}, m, \mathrm{H}-2, \mathrm{H}-23)$.

\section{Margaric acid, (6)}

The fraction $\mathrm{CC} 1-\mathrm{F}_{10}(1.84 \mathrm{~g})$ was fractionated using Sephadex LH-20 column chromatography with $1 \%$ methanol in chloroform to yield ten fractions. The fourth fraction was then eluted using silica gel column chromatography with chloroform-ethyl acetate (4:1) to obtain ten more fractions. The fourth fraction was further purified using silica gel column chromatography 


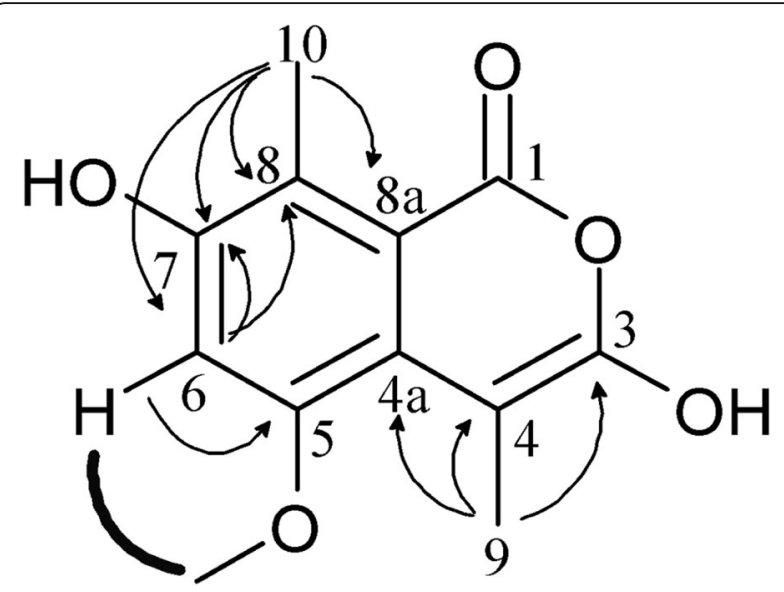

Fig. $2 \mathrm{HMBC}(\mathrm{H} \rightarrow \mathrm{C})$ and $\operatorname{COSY}(\mathrm{H} \quad \mathrm{H})$ correlations for compound 1 obtained using NMR spectrometric technique

with chloroform-ethyl acetate (3:1) to obtain compound 6. Compound $\mathbf{6}$ was obtained as a white amorphous solid $(9.0 \mathrm{mg})$, and the data for its structural elucidation were as follows: $\mathrm{UV}(\mathrm{EtOH}) \lambda_{\max } \mathrm{nm}(\log \varepsilon): 202$ (2.47); IR (ATR) $\max , \mathrm{cm}^{-1}: 2916,2848,1706,1697,1463$, 1430, 1411, 1310, 1295, 1272, 1251, 1229, 1208, 1188, 939, and 720; EI-MS $m / z: 269[\mathrm{M}-1]^{+}$(calculated for $\left.\mathrm{C}_{17} \mathrm{H}_{34} \mathrm{O}_{2}, 270.0 \mathrm{~g} / \mathrm{mol}\right) ;{ }^{1} \mathrm{H}-\mathrm{NMR}\left(\mathrm{CDCl}_{3}, 600 \mathrm{MHz}\right) \delta_{\mathrm{H}}$ (ppm): 0.89 (3H, $t, J=7.2, \mathrm{H}-17), 1.26-1.32(26 \mathrm{H}, m, \mathrm{H}-$ $4-\mathrm{H}-16$, overlapped $), 1.65(2 \mathrm{H}, m, \mathrm{H}-3)$, and $2.35(2 \mathrm{H}, t$, $J=7.5 \mathrm{~Hz}, \mathrm{H}-2) ;{ }^{13} \mathrm{C}-\mathrm{NMR}\left(\mathrm{CDCl}_{3}, 150 \mathrm{MHz}\right) \delta_{\mathrm{C}}(\mathrm{ppm})$ : 14.2 (C-17), 22.7 (C-16), 24.7 (C-3), 29.1-31.9 (C-4-C15), 34.1 (C-2), and $180.3(\mathrm{C}-1)$.

Table $3^{1} \mathrm{H}$ - and ${ }^{13} \mathrm{C}-\mathrm{NMR}$ spectra data for compound $\mathbf{1}$

\begin{tabular}{lll}
\hline No & $\boldsymbol{\delta}_{\mathbf{C}}(\mathbf{p p m})$ & $\boldsymbol{\delta}_{\mathbf{H}}(\mathbf{p p m})$ \\
\hline 1 & 162.7 & - \\
3 & 157.1 & - \\
4 & 139.9 & - \\
$4 \mathrm{a}$ & 110.2 & - \\
5 & 153.1 & - \\
$\mathrm{OCH}_{3}$ & 56.6 & $3.89, \mathrm{~s}$ \\
6 & 101.8 & $6.91, \mathrm{~s}$ \\
7 & 142.5 & - \\
$\mathrm{OH}$ & - & - \\
8 & 119.8 & - \\
$8 \mathrm{a}$ & 131.1 & \\
9 & 18.8 & $2.45, \mathrm{~s}$ \\
10 & 15.2 & $2.65, \mathrm{~s}$ \\
\hline
\end{tabular}

\section{Statistical analysis}

Assay data obtained were subjected to one-way ANOVA with post-hoc Tukey's multiple comparisons test using GraphPad Prism 5 software (La Jolla, CA, USA). The data are expressed as mean \pm standard error of the mean (S.E.M.) with triplicate measurements $(n=3)$. The difference between means was determined at $95 \%$ confidence intervals, with $p$ value $<0.05$ considered as significantly different.

\section{Results}

In vitro xanthine oxidase inhibitory activity of $M$. pumilum varieties

Among the eighteen extracts assayed, five exhibited more than $50 \%$ xanthine oxidase inhibition, and their inhibitions were less than that of the positive control, allopurinol $\left(99.82 \pm 0.00 \%, \quad \mathrm{IC}_{50}=0.24 \pm 0.00 \mu \mathrm{g} / \mathrm{mL}\right)$. They were the dichloromethane extracts of var. alata $\left(68.21 \pm 2.50 \%, \mathrm{IC}_{50}=310.9 \pm 8.25 \mu \mathrm{g} / \mathrm{mL}\right)$, var. pumila $\left(85.77 \pm 0.70 \%, \quad \mathrm{IC}_{50}=161.6 \pm 7.35 \mu \mathrm{g} / \mathrm{mL}\right), \quad$ and var. lanceolata $\left(74.33 \pm 4.33 \%, \quad \mathrm{IC}_{50}=233.1 \pm 19.85 \mu \mathrm{g} / \mathrm{mL}\right)$ leaves, and the methanol extracts of var. pumila $\left(80.97 \pm 0.72 \%, \mathrm{IC}_{50}=175.1 \pm 0.20 \mu \mathrm{g} / \mathrm{mL}\right)$ and var. lanceolata $\quad\left(67.52 \pm 0.35 \%, \quad \mathrm{IC}_{50}=185.3 \pm 2.50 \mu \mathrm{g} / \mathrm{mL}\right)$ leaves (Tables 1 and 2). The dichloromethane extract of var. pumila leaves was considered to be more active than the other extracts because it had the highest percentage of xanthine oxidase inhibition and the lowest $\mathrm{IC}_{50}$ value. Thus, the extract was subjected to further fractionation processes that led to the isolation of six pure compounds.

Structural elucidation of compounds isolated from the dichloromethane extract of $M$. pumilum var. pumila leaves and their xanthine oxidase inhibitory activity

Compound 1 was obtained as a white amorphous powder from the dichloromethane fraction, and its molecular formula was established as 7 degrees of unsaturation. Its UV spectrum showed maximum absorption at 275 nm. Its ATR-FTIR spectrum showed strong absorption at $3256(\mathrm{O}-\mathrm{H}$ stretching), $1734(\mathrm{C}=\mathrm{O}), 1473-1424(\mathrm{C}-\mathrm{H}$ bending), and 1256 (C-O stretching) $\mathrm{cm}^{-1}$. Its ${ }^{1} \mathrm{H}-\mathrm{NMR}$ spectrum $\left(\mathrm{CDCl}_{3}, 600 \mathrm{MHz}\right)$ showed methyl protons at $\delta 2.45(3 \mathrm{H}, \mathrm{H}-9)$ and $\delta 2.65(3 \mathrm{H}, s, \mathrm{H}-10)$, a methoxy proton at $\delta 3.89(3 \mathrm{H}, s, \mathrm{H}-5)$, and an aromatic proton at $\delta 6.91(s)$. Its ${ }^{13} \mathrm{C}$-NMR spectrum $\left(\mathrm{CDCl}_{3}, 150 \mathrm{MHz}\right)$ showed 12 carbons with signals indicating the presence of one carbonyl carbon at $\delta 162.7(\mathrm{C}-1)$, one methoxy carbon at $\delta 153.1(\mathrm{C}-5)$, and two methyl carbons at $\delta$ $18.8 \quad(\mathrm{C}-11)$ and $\delta \quad 15.2 \quad(\mathrm{C}-12)$. Its ${ }^{1} \mathrm{H}-{ }^{1} \mathrm{H} \quad \mathrm{COSY}$ spectrum displayed the correlation between aromatic protons at $\delta 6.91(s)$ and methoxy protons at $\delta 3.89$, thus revealing the location of the aromatic proton group at the C-6 position. Its HSQC spectrum showed 


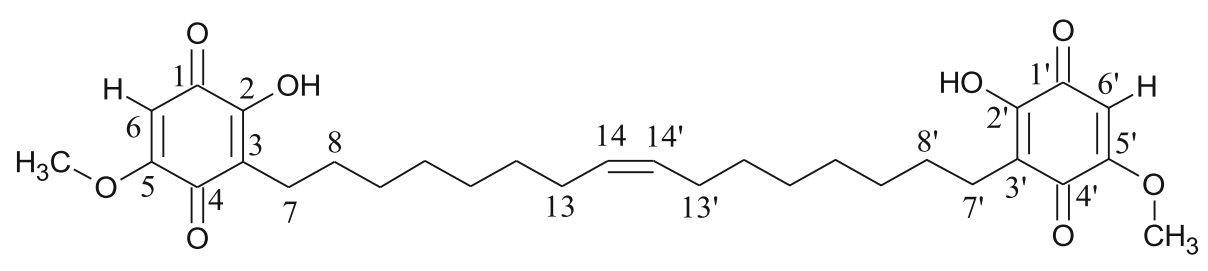

Ardisiaquinone A (2)

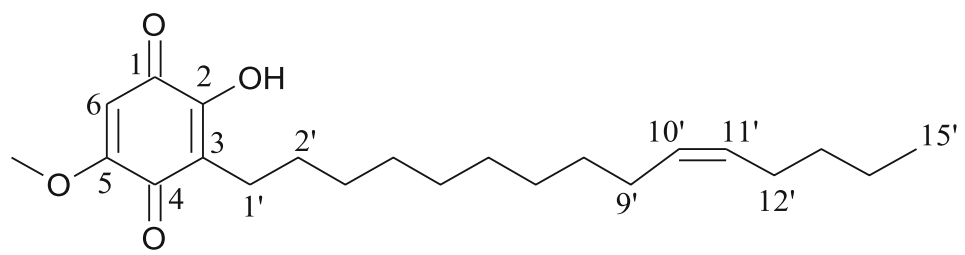

Maesanin (3)

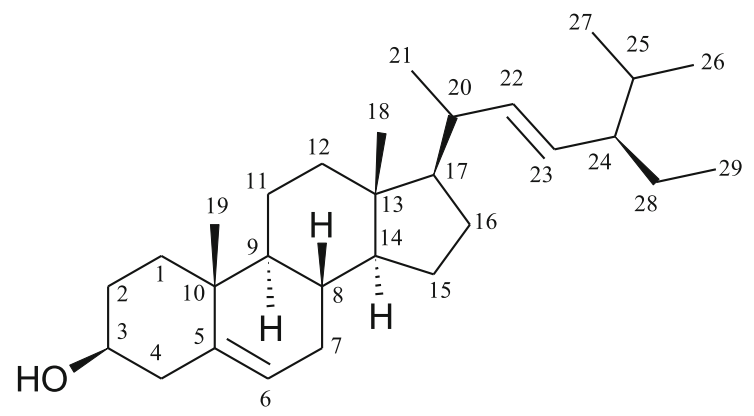

Stigmasterol (4)

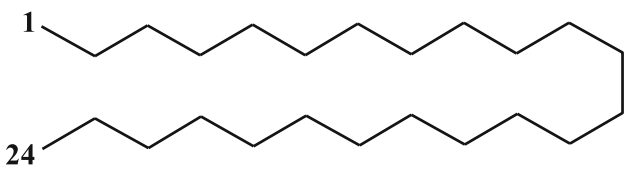

Tetracosane (5)<smiles>[3H]CCCCCCCCCCCCCCCC(=O)O</smiles>

Margaric acid (6)

Fig. 3 Chemical structures of compounds $\mathbf{2}-\mathbf{6}$ elucidated using UV, IR, MS, and NMR spectroscopic techniques

correlations between methoxy protons at $\delta 3.89$ and $\delta$ 56.6 (C-5), an aromatic proton at $\delta 6.9$ and $\delta 101.8$ (C6), and two methyl protons at $\delta 2.45$ and $\delta 2.65$ and $\delta$ $18.8(C-9)$ and $\delta 15.2(C-10)$, respectively. In its HMBC spectrum, the linkage of two methyl protons was established by the cross peaks between H-9 ( $\delta$ 2.45) and C-4 $\left(\delta 139.9,{ }^{2}\right)$ ), C-4a $\left(\delta 110.2,{ }^{3}\right)$ ), and C-3 $\left.\left(\delta 157.1,{ }^{3}\right)\right)$, and between $\mathrm{H}-10(\delta 2.65)$ and $\left.\mathrm{C}-7\left(\delta 142.5,{ }^{3}\right)\right), \mathrm{C}-8(\delta$ $\left.119.8,{ }^{2} \gamma\right)$, and $\mathrm{C}-8 \mathrm{a}\left(\delta 131.1,{ }^{3} \gamma\right)$. This correlation confirmed the position of methyl protons in compound $\mathbf{1}$. The spectrum also showed the correlation between methoxy protons at $\delta 3.89$ and $C-5\left(\delta 153.1,{ }^{2}\right)$, and the 
correlation between aromatic protons $(\delta$ 6.91) and C-5 $\left(\delta 153.1,{ }^{2} \gamma\right), \mathrm{C}-7\left(\delta 142.5,{ }^{2} \gamma\right)$, and C-8 ( $\left.\delta 119.8,{ }^{3}\right)$ ). Therefore, based on the data above the structure of compound 1 was determined as 3,7-dihydroxy-5-methoxy-4,8-dimethyl-isocoumarin (Fig. 2). All ${ }^{1} \mathrm{H}-\mathrm{NMR}$ and ${ }^{13} \mathrm{C}$-NMR data of compound $\mathbf{1}$ are shown in Table 3.

The other five known compounds were identified as ardisiaquinone A (2) [41], maesanin (3) [42], stigmasterol (4) [43], tetracosane (5) [44], and margaric acid (6) [45] (Fig. 3) by comparing MS and NMR data with those reported in the literature.

Of the six compounds, only 3,7-dihydroxy-5-methoxy4,8-dimethyl-isocoumarin (1) and ardisiaquinone A (2) exhibited more than $50 \%$ xanthine oxidase inhibition (Table 4), with the former (1) being more potent than the latter (2). The former (1) had an $\mathrm{IC}_{50}$ value of $0.66 \pm$ $0.01 \mu \mathrm{g} / \mathrm{mL}$, which is comparable $(p>0.05)$ with that of allopurinol $\left(\mathrm{IC}_{50}=0.24 \pm 0.00 \mu \mathrm{g} / \mathrm{mL}\right)$ (Table 5).

\section{Discussion}

The extract of M. pumilum var. pumila leaves inhibited xanthine oxidase in vitro. The findings of this study support the previous report [39] in which ethanol (80\%) $M$. pumilum var. pumila leaf extract showed antihyperuricemic effect by inhibiting hepatic xanthine oxidase and reducing serum uric acid levels in hyperuricemic-induced male Sprague-Dawley rats 14 days after treatment with $200 \mathrm{mg} / \mathrm{kg}$ extract.

In this study, a new compound (3,7-dihydroxy-5-methoxy4,8-dimethyl-isocoumarin) was isolated from the dichloromethane extract of $M$. pumilum var. pumila leaves, which was found to be the most active extract $\left(\mathrm{IC}_{50}=161.6 \pm\right.$ $7.35 \mu \mathrm{g} / \mathrm{mL})$. The compound had an $\mathrm{IC}_{50}$ value $(0.66 \pm$ $0.01 \mu \mathrm{g} / \mathrm{mL}$ ) that was comparable to that of allopurinol $\left(\mathrm{IC}_{50}=0.24 \pm 0.00 \mu \mathrm{g} / \mathrm{mL}\right)$ and could be a potential xanthine oxidase inhibitor. A study by Lin et al. [46] demonstrated competitive inhibition of selected coumarins (e.g., coumarin, 4-hydroxycoumarin, 7-hydroxycoumarin, esculetin, scopoletin, dihydrocoumarin, and 7-hydroxy-4-methylesculetin) against xanthine oxidase. Esculetin was found to be the most potent inhibitor through substrate binding blockade. It was suggested that the two hydroxyl moieties on its benzene ring contributed to its activity by forming hydrogen bonds with the active site of xanthine oxidase. Therefore, the presence of two hydroxyl groups in the structure of 3,7-dihydroxy-5methoxy-4,8-dimethyl-isocoumarin (1) could explain the basis of its xanthine oxidase inhibitory activity. Another study also reported that the xanthine oxidase inhibitory activity of 5,7-dihydroxy-3-(3-hydroxyphenyl) coumarin was 7fold better than that of allopurinol [47]. The low activity of ardisiaquinone A (2) and lack of activity of the other isolated compounds (3-6) obtained in this study could be explained by the difference in molecular structure that influences the stability of hydrophilic and hydrophobic characteristics on the xanthine oxidase active binding site [48].

There are several reviews on the anti-hyperuricemic effects of foods [49], Chinese herbs [50], and natural products [51]. Hyperuricemia has been linked with cardiovascular disease, hypertension, diabetes, obesity, chronic kidney disease, and many other diseases [52, 53]. Its prevalence in the female population and postmenopausal women has been reported [54-56]. The data from the Third National Health and Nutrition Examination Survey showed that menopause was associated with higher serum uric acid levels and postmenopausal hormone replacement was associated with lower serum uric acid levels, suggesting that estrogen plays a key role in protecting women from hyperuricemia and gout [57]. Several publications have reported on the potential use of M. pumilum extract to alleviate postmenopausal conditions due to estrogenic properties [58-60], hypercholesterolemia [61], and hypertension [62]. Thus, the extract of M. pumilum var. pumilum could be beneficial in preventing or treating hyperuricemic-related diseases, while 3,7-dihydroxy-5-methoxy-4,8-dimethylisocoumarin (1) could be used as an analytical marker to standardize the extract and formulated herbal products. Standardization by simultaneous quantification of xanthine oxidase inhibitors from Zanthoxylum armatum fruits using high-performance liquid

Table 4 Percentages of xanthine oxidase inhibition of isolated compounds and allopurinol

\begin{tabular}{ll}
\hline Compound & Percentage of xanthine oxidase inhibition (\%) $^{\mathbf{a}}$ \\
\hline $\mathbf{1}$ & $98.46 \pm 0.37$ \\
$\mathbf{3}$ & $91.54 \pm 0.08$ \\
$\mathbf{4}$ & 0.00 \\
$\mathbf{5}$ & 0.00 \\
$\mathbf{6}$ & 0.00 \\
Allopurinol (positive control) & 0.00 \\
\hline
\end{tabular}

Data are presented as mean \pm S.E.M. of three replicates $(n=3)$

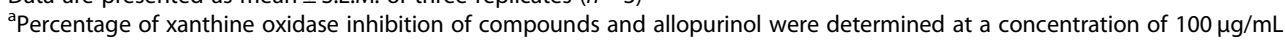


Table $\mathbf{5} I C_{50}$ values of xanthine oxidase inhibition of compounds $\mathbf{1}$ and $\mathbf{2}$ compared to allopurinol

\begin{tabular}{|c|c|c|c|}
\hline Compound & $\begin{array}{l}\text { Concentration }(\mu \mathrm{g} / \\
\mathrm{mL})\end{array}$ & $\begin{array}{l}\text { Percentage of xanthine oxidase inhibition } \\
\text { (\%) }\end{array}$ & $\begin{array}{l}\mathrm{IC}_{50} \text { value of xanthine oxidase inhibition }(\mu \mathrm{g} / \\
\mathrm{mL})^{\mathrm{a}}\end{array}$ \\
\hline \multirow[t]{9}{*}{1} & 0.39 & $39.57 \pm 0.68$ & $0.66 \pm 0.01^{\mathrm{b}}$ \\
\hline & 0.78 & $54.14 \pm 0.34$ & \\
\hline & 1.56 & $66.49 \pm 0.05$ & \\
\hline & 3.13 & $76.17 \pm 0.52$ & \\
\hline & 6.25 & $86.02 \pm 0.14$ & \\
\hline & 12.5 & $91.75 \pm 0.22$ & \\
\hline & 25 & $95.04 \pm 0.86$ & \\
\hline & 50 & $96.91 \pm 0.06$ & \\
\hline & 100 & $98.46 \pm 0.37$ & \\
\hline \multirow[t]{5}{*}{2} & 6.25 & $9.11 \pm 0.28$ & $31.2 \pm 1.28$ \\
\hline & 12.5 & $14.54 \pm 0.45$ & \\
\hline & 25 & $36.31 \pm 0.08$ & \\
\hline & 50 & $74.08 \pm 0.50$ & \\
\hline & 100 & $91.54 \pm 0.08$ & \\
\hline \multirow{7}{*}{$\begin{array}{l}\text { Allopurinol (positive } \\
\text { control) }\end{array}$} & 0.0064 & $4.12 \pm 0.00$ & $0.24 \pm 0.00$ \\
\hline & 0.032 & $17.81 \pm 0.00$ & \\
\hline & 0.16 & $29.47 \pm 0.00$ & \\
\hline & 0.8 & $93.66 \pm 0.00$ & \\
\hline & 4 & $98.86 \pm 0.00$ & \\
\hline & 20 & $99.01 \pm 0.00$ & \\
\hline & 100 & $99.82 \pm 0.00$ & \\
\hline
\end{tabular}

Data are presented as mean \pm S.E.M. of three replicates $(n=3)$

${ }^{\mathrm{a}} \mathrm{I} \mathrm{C}_{50}$ values were obtained based on the percentage of xanthine oxidase inhibition of compounds and allopurinol at different concentrations using the Graphpad Prism 5 software (La Jolla, CA, USA)

${ }^{\mathrm{b}}$ Not significantly different compared to allopurinol $(p>0.05)$, analyzed using one-way ANOVA followed by Tukey's multiple comparisons test

chromatography with a photometric diode array detector (HPLC-PDA) has been reported [63].

\section{Conclusions}

In the present study, three varieties of M. pumilum were investigated based on their ethnomedical uses and biological activities. The study identified a new isocoumarin compound, 3,7-dihydroxy-5-methoxy-4,8-dimethyl-isocoumarin (1), from the dichloromethane extract of $M$. pumilum var. pumila leaves. The compound was the most active xanthine oxidase inhibitor and had an $\mathrm{IC}_{50}$ value $(0.66 \pm 0.01 \mu \mathrm{g} / \mathrm{mL})$ that was comparable with that of allopurinol $\left(\mathrm{IC}_{50}=0.24 \pm 0.00 \mu \mathrm{g} / \mathrm{mL}\right)$. Therefore, $M$. pumilum var. pumila leaves could potentially be a source of new natural xanthine oxidase inhibitors. However, in vivo studies are required to establish its efficacy and safety.

\section{Abbreviations}

$\mathrm{IC}_{50}$ : The half maximal inhibitory concentration; HPTLC: High performance thin later chromatography; HPLC: High performance liquid chromatography; ATR-FTIR: Attenuated total reflectance-fourier transform infrared spectroscopy; XO: Xanthine oxidase; VLC: Vacuum liquid chromatography;
CC: Column chromatography; TLC: Thin layer chromatography; UV: Ultraviolet spectrophotometry; IR: Infrared spectrophotometry; NMR: Nuclear magnetic resonance spectroscopy; HR-ESI-MS: High-resolution electrospray ionization mass spectrometry; El-MS: Electron ionization mass spectrometry; DCM: Dichloromethane; $\mathrm{MeOH}$ : Methanol; $\mathrm{CHCl}_{3}$ : Chloroform; DMSO: Dimethyl sulfoxide; EtOAc: Ethyl acetate; EtOH: Ethanol; $\mathrm{HCl}$ : Hydrochloric acid; $\mathrm{NaOH}$ : Sodium hydroxide; $\mathrm{KH}_{2} \mathrm{PO}_{4}$ : Potassium dihydrogen phosphate; UKMB: Herbarium of Universiti Kebangsaan Malaysia in Bangi; $\lambda_{\text {max }}$ : Wavelength of maximum absorbance; $\log \varepsilon$ : Log of molar absorption coefficient; $U_{\max }$ : Maximum frequency; $m / z$ : Mass-to-charge ratio; ${ }^{1} \mathrm{H}$-NMR: Proton nuclear magnetic resonance; $\mathrm{CDCl}_{3}$ : Deuterated chloroform; $\delta_{H}$ : Proton chemical shift; s: Singlet; ${ }^{13} \mathrm{C}-\mathrm{NMR}$ : Carbon-13 nuclear magnetic resonance; $\delta_{c}$ : Carbon chemical shift; $m$ : Multiplet; $d$ : Doublet; J: Coupling constant; br: Broad; $\mathrm{CD}_{3} \mathrm{OD}$ : Deuterated methanol; $t$ : Triplet; $d d$ : Double doublet; S.E.M.: Standard error of the mean; n: Number; ANOVA: Analysis of variance; $p$ : Probability; COSY: Correlated spectroscopy; HSQC: Heteronuclear single quantum coherence spectroscopy; HMBC: Heteronuclear multiple bond correlation spectroscopy; HPLC-PDA: High performance liquid chromatography-photometric diode array

\section{Acknowledgments}

We would like to thank the Ministry of Agriculture and Agro-Based Industry, Malaysia (NRGS/NH0711D002) and UKM Zamalah (2011/2012) for financial support; Mr. Sani Miran ${ }^{\dagger}$ (UKM Herbarium) for plant materials collection and identification; the Center for Research and Instrumentation (CRIM), Universiti Kebangsaan Malaysia for NMR and ESIMS analyses; staff of the Faculty of Pharmacy, Universiti Kebangsaan Malaysia for technical assistance; and Editage (www.editage.com) for English language editing. 


\section{Authors' contributions}

$\mathrm{JAJ}, \mathrm{KH}$ and $\mathrm{JJ}$ designed the study and revised the manuscript. NAA performed all experiments, analysed the data and drafted the manuscript. CWS helped with some data analysis. All authors have read and approved the manuscript.

\section{Funding}

The project was funded by the Ministry of Agriculture and Agro-Based Industry, Malaysia with a research grant number NRGS/NH0711D002 and UKM Zamalah (2011/2012) for financial support. The funding bodies did not contribute to study design, data collection, analysis, interpretation and manuscript writing.

\section{Availability of data and materials}

All relevant data regarding the study is included in this article and any supplementary data is available from the corresponding author upon request.

\section{Ethics approval and consent to participate}

Not applicable.

\section{Consent for publication}

Not applicable.

\section{Competing interests}

The authors declare that they have no conflict of interests.

\section{Author details}

${ }^{1}$ Drug and Herbal Research Centre, Faculty of Pharmacy, Universiti Kebangsaan Malaysia, Kuala Lumpur, Malaysia. ${ }^{2}$ Department of Pharmacy, Faculty of Science and Technology, Universitas Sembilanbelas November Kolaka, Kolaka, Indonesia.

Received: 15 April 2020 Accepted: 19 October 2020

Published online: 27 October 2020

\section{References}

1. The Plant List. Version 1.1. Published on the Internet; http://www. theplantlist.org/ (Accessed 6 April 2020). 2013.

2. Stone BC. New and noteworthy Malesian Myrsinaceae III. On the genus Ardisia Sw. Borneo Proc Acad Nat Sci Philadelphia. 1989;141:263-306.

3. Sunarno B. Revision of the genus Labisia (Myrsinaceae). Blumea. 2005;50(3) 579-97.

4. Aladdin NA, Jamal JA, Talip N, Hamsani NAM, Rahman MRA, Sabandar CW, Muhammad K, Husain K, Jalil J. Comparative study of three Marantodes pumilum varieties by microscopy, spectroscopy and chromatography. Rev Bras Farmacogn. 2016:26(1):1-14.

5. Burkill $\Perp H$. Dictionary of the Economic Products of the Malay Peninsula. Crown Agents of the Colonies, London, England. Vol. 2, 1935;1290.

6. Zakaria M, Mohd MA. Traditional Malay medicinal plants. Kuala Lumpur: Fajar Bakti; 1994.

7. Quattrocchi U. CRC world dictionary of medicinal and poisonous plants: common names, Scientific Names, Eponyms, Synonyms and Etymology. Roca Baton: CRC Press; 2012.

8. Runi SP. Studies on medicinal plant in Sarawak. In the seminar on medicinal and aromatic plants, Kuala Lumpur (Malaysia), 12-13 Sep 2000. Forest Research Institute Malaysia (FRIM); 2000

9. Singh GD, Ganjoo M, Youssouf MS, Koul A, Sharma R, Singh S, Sangwan PL, Koul S, Ahamad DB, Johri RK. Sub-acute toxicity evaluation of an aqueous extract of Labisia pumila, a Malaysian herb. Food Chem Toxicol. 2009;47(10):2661-5.

10. Norhaiza M, Maziah M, Hakiman M. Antioxidative properties of leaf extracts of a popular Malaysian herb Labisia pumila. J Med Plant Res. 2009:3:217-23.

11. Mamat N, Jamal JA, Jantan I, Husain K. Xanthine oxidase inhibitory and DPPH radical scavenging activities of some Primulaceae species. Sains Malays. 2014;43(12):1827-33.

12. Khairul FK, Nurul HM, Zhari I. Antioxidative properties of various extract of Labisia pumila (Kacip Fatimah). Curr Trends Perspect. 2005:4:306-12.

13. Karimi E, Jaafar HZ, Ahmad S. Phytochemical analysis and antimicrobial activities of methanolic extracts of leaf, stem and root from different varieties of Labisia pumila Benth. Molecules. 2011;16(6):4438-50.
14. Rahmi EP, Jamal JA, Kumolosasi E, Jalil J, Aladdin NA. Marantodes pumilum (Blume) Kuntze inhibited secretion of lipopolysaccharide- and monosodium urate crystal-stimulated cytokines and plasma prostaglandin $E_{2}$. Pharmacogn Mag. 2017;13(Suppl. 3):S578-86

15. Wan Omar WFN, Giribabu N, Karim K, Salleh N. Marantodes pumilum (Blume) Kuntze (Kacip Fatimah) stimulates uterine contraction in rats in post-partum period. J Ethnopharmacol. 2019;245:112175

16. Jamal JA, Ramli N, Stanslas J, Husain K. Estrogenic activity of selected Myrsinaceae species in MCF-7 human breast cancer cells. Int J Pharm Pharm Sci. 2012;4:547-53.

17. Pandey A, Bani S, Sangwan PL. Anti-obesity potential of gallic acid from Labisia pumila, through augmentation of adipokines in high fat diet induced obesity in C57BL/6 mice. Adv Res. 2014:2(10):556-70.

18. Choi H, Kim D, Kim JW, Ngadiran S, Sarmidi MR, Park CS. Labisia pumila extract protects skin cells from photoageing caused by UVB irradiation. J Biosci Bioeng. 2010;109:291-6.

19. Pihie AHL, Othman F, Zakaria ZA. Anticarcinogenic activity of Labisia pumila against 7,12-dimethylbenz(a) anthracene (DMBA)/croton oil-induced mouse skin carcinogenesis. Afr J Pharm Pharmaco. 2011:5:823-32.

20. Ali Z, Khan IA. Alkyl phenols and saponins from the roots of Labisia pumila (Kacip Fatimah). Phytochemistry. 2011;72:2075-80

21. Al-Mekhlafi NA, Shaari K, Abas F, Kneer R, Jeyaraj EJ, Stanslas J, Yamamoto N Honda T, Lajis NH. Alkenylresorcinols and cytotoxic activity of the constituents isolated from Labisia pumila. Phytochemistry. 2012;80:42-9.

22. Avula B, Wang YH, Ali Z, Smillie TJ, Khan IA. Quantitative determination of triperpene saponins and alkenated-phenolics from Labisia pumila using an LC-UV/ELSD method and confirmation by LC-ESI-TOF. Planta Med. 2011; 77(15):1742-8.

23. Karimi E, Jaafar HZ, Ghasemzadeh A, Ebrahimi M. Fatty acid composition, antioxidant and antibacterial properties of the microwave aqueous extract of three varieties of Labisia pumila Benth. Biol Res. 2015;48(1):9.

24. Stasiuk M, Kozubek A. Biological activity of phenolic lipids. Cell Mol Life Sci. 2010;67(6):841-60

25. Chiang HC, Lo YJ, Lu FJ. Xanthine oxidase inhibitors from leaves of Alsophila spinulosa (hook) Tryon. J Enzym Inhib. 1994;8:61-71.

26. Cos $P$, Ying $L$, Calomme M, Hu JP, Cimanga $K$, Van Poel B, Pieters L, Vlietinck AJ, Berghe DV. Structure-activity relationship and classification of flavonoids as inhibitors of xanthine oxidase and superoxide scavengers. J Nat Prod. 1998;61:71-6.

27. Pacher P, Nivorozhkin A, Szabó C. Therapeutic effects of xanthine oxidase inhibitors: renaissance half a century after the discovery of allopurinol. Pharmacol Rev. 2006;58(1):87-114.

28. Rasaratnam I, Christophidis N. Gout: 'a disease of plenty'. Aust Fam Physician. 1995;24:849-60.

29. Stack A, Manolis AJ, Ritz E. Detrimental role of hyperuricemia on the cardioReno-vascular system. Curr Med Res Opin. 2015;31(Suppl. 2):21-6.

30. Johnson RJ, Kivlighn SD, Kim YG, Suga S, Fogo AB. Reappraisal of the pathogenesis and consequences of hyperuricemia in hypertension, cardiovascular disease, and renal disease. Am J Kidney Dis. 1999:33(2):225-34

31. Ogino K, Hisatome I, Shigemasa C. Anti-hyperuricemic agents. Nihon Rinsho. 2003:61(1):197-201.

32. Unno T, Sugimoto A, Kakuda T. Xanthine oxidase inhibitors from the leaves of Lagerstroemia speciosa (L.) Pers. J. Ethnopharmacol. 2004;93(2):391-5.

33. Tapsell LC, Hemphill I, Cobiac L, Sullivan DR, Fenech M, Patch CS, Roodenrys S, Keogh JB, Clifton PM, Williams PG, Fazio VA, Inge KE. Health benefits of herbs and spices: the past, the present, the future. Med J Aust. 2006;185(4):S1-S24.

34. Costantino L, Albasini A, Rastelli G, Benvenuti S. Activity of polyphenolic crude extracts as scavengers of superoxide radicals and inhibitors of xanthine oxidase. Planta Med. 1992;58:342-4.

35. lio M, Moriyama A, Matsumoto Y, Takaki N, Fukumoto M. Inhibition of xanthine oxidase by flavonoids by folate compounds and amethopterin. J Biol Chem. 1985:259:12-5.

36. Liu HX, He MT, Tan HB, Gu W, Yang SX, Wang YH, Li L, Long CL. Xanthine oxidase inhibitors isolated from Piper nudibaccatum. Phytochem Lett. 2015;12:133-7.

37. Chang WS, Chiang HC. Structure-activity relationship of coumarins in xanthine oxidase inhibition. Anticancer Res. 1995:15(5B):1969-73.

38. Zuo J, Zhang W, Jian H, Bou-Chacra N, Löbenberg R. Esculetin as bioactive marker: towards a rational scientific approach for the treatment of hyperuricemia using traditional Chinese medicine. Braz J Pharm Sci. 2020;56:e17827.

39. Rahmi EP, Kumolosasi E, Jalil J, Husain K, Buang F, Abd. Razak AF, Jamal JA Anti-hyperuricemic and anti-inflammatory effects of Marantodes pumilum as 
potential treatment for gout. Front Pharmacol. 2020. https://doi.org/10. 3389/fphar.2020.00289.

40. Seruji NMU, Khong HY, Kutoi CJ. Antioxidant, anti-inflammatory, and cytotoxic activities of Garcinia nervosa (Clusiaceae). J Chem. 2013;2012:1-5.

41. Fukuyama Y, Kriryama Y, Kodama M, Iwaki H, Hosozawa S, Aki S, Matsui K. Naturally occuring 5-lipoxygenase inhibitors. (M. 1) structures of ardisiaquinones D, E, and F from Ardisiasieboldii. Chem Pharm Bull. 1995;43(8):1391-4.

42. Yan-Fang L, Jia L, Qiang S, Li-Hong H. Benzoquinones from Ardisia japonica with inhibitory activity towards human protein tyrosine phosphatase $1 \mathrm{~B}$ (PTP1B). Chem Biodivers. 2007:4:961-5.

43. Forgo P, Kövér KE. Gradient enhanced selective experiments in the ${ }^{1} H$ NMR chemical shift assignment of the skeleton and side-chain resonances of stigmasterol, a phytosterol derivative. Steroid. 2004;69:43-50.

44. Kalegari M, Miguel MD, Dias JFG, Lordello ALL, Lima CP, Miyazaki CMS, Zanin SMW, Verdam MCS, Miguel OG. Phytochemical constituents and preliminary toxicity evaluation of leaves from Rourea induta planch. (Connaraceae). Braz. J Pharm Sci. 2011:47(3):635-42.

45. The Human Metabolome Database (HMDB). Showing metabocard for heptadecanoic acid (HMDB02259). 2009; http://www.hmdb.ca/metabolites/ hmdb02259\#spectra.

46. Lin HC, Tsai SH, Chen CS, Chang YC, Lee CM, Lai ZY, Lin CM. Structure-activity relationship of coumarin derivatives on xanthine oxidase-inhibiting and free radical-scavenging activities. Biochem Pharmacol. 2008;75(6):1416-25.

47. Fais A, Era B, Asthana S, Sogos V, Medda R, Santana L, Uriarte E, Matos MJ, Delogu F, Kumar A. Coumarin derivatives as promising xanthine oxidase inhibitors. Int J Biol Macromol. 2018;120(A):1286-93.

48. Masuoka N, Nihei K, Masuoka T, Kuroda K, Sasaki K, Kubo I. The inhibition of uric acid formation catalyzed by xanthine oxidase properties of the alkyl caffeates and cardol. J Food Res. 2012;1(3):257-62.

49. Luxita S, Zarrin A. The role of xanthine oxidase inhibiting foods in therapeutic management of hyperuricemia and gout. Int J Res Soc Sci. 2018;8(11):253-67.

50. Lin J, Chen S, Li S, Lu M, Li Y, Su Y. Efficacy and safety of Chinese medicinal herbs for the treatment of hyperuricemia: a systematic review and metaanalysis. Evid Based Complement Altern Med. 2016;2016:2146204.

51. El-Tantawy WH. Natural products for the management of hyperuricaemia and gout: a review. Arch Physiol Biochem. 2019. https://doi.org/10.1080/ 13813455.2019.1610779.

52. Becker MA, Jolly M. Hyperuricemia and associated diseases. Rheum Dis Clin N Am. 2006;32:275-93.

53. Ali N, Mahmood S, Islam F, Rahman S, Haque T, Islam S, Rahman S, Haque N, Mosaib G, Perveen R, Jhanum FA. Relationship between serum uric acid and hypertension: a cross-sectional study in Bangladeshi adults. Sci Rep. 2019;9:9061.

54. Liu B, Wang T, Zhao HN, Yue WW, Yu HP, Liu CX, Yin J, Jia RY, Nie HW. The prevalence of hyperuricemia in China: a meta-analysis. BMC Public Health. 2011;11:823.

55. Ali N, Perveen R, Rahman S, Mahmood S, Rahman S, Islam S, Haque T, Sumon AH, Kathak RR, Molla NH, Islam F, Mohanto NC, Nurunnabi SM, Ahmed S, Rahman M. Prevalence of hyperuricemia and the relationship between serum uric acid and obesity: a study on Bangladeshi adults. PLoS One. 2018;13(11):e0206850

56. Guan S, Tang Z, Fang X, Wu X, Liu H, Wang C, Hou C. Prevalence of hyperuricemia among Beijing post-menopausal women in 10 years. Arch Gerontol Geriat. 2016;64:162-6.

57. Hak EA, Choi HK. Menopause, postmenopausal hormone use and serum uric acid levels in US women - the third national health and nutrition examination survey. Arthritis Res Ther. 2008;10:R116.

58. Hairi HA, Mohd Sofi NS, Khodari SN, Jamal JA, Mohamed IN, Shuid AN. Therapeutic effects of Labisia pumila on estrogen-deficiency related disorders: an evidence-based review. Int J Pharmacol. 2016;12(5):451-60.

59. Giaze TR, Shuid AN, Soelaiman IN, Muhammad N, Jamal JA, Fauzi MB, Mohamed N. Comparative anti-osteoporotic properties of the leaves and roots of Marantodes pumilum var. alata in postmenopausal rat model. J Tradit Complement Med. 2019:9(4):393-400.

60. Dharmani M, Kamarulzaman K, Giribabu N, Choy KW, Aladdin NA, Jamal JA, Mustafa MR. Effect of Marantodes pumilum Blume (Kuntze) var. alata on $\beta$ cell function and insulin signalling in ovariectomised diabetic rats. Phytomedicine. 2019;65:153101.

61. Dianita R, Jantan I, Jalil J, Amran AZ. Effects of Labisia pumila var. alata extracts on the lipid profile, serum antioxidant status and abdominal aorta of high-cholesterol diet rats. Phytomedicine. 2016;23(8):810-7.

62. Manshor NM, Razali N, Jusoh RR, Asmawi MZ, Mohamed N, Zainol S, Altaf E, Dewa A. Vasorelaxant effect of water fraction of Labisia pumila and its mechanisms in spontaneously hypertensive rats aortic ring preparation. Int $J$ Cardiol Hypertens. 2020;4:100024.

63. Rajana, Nooreen Z, Bushra U, Jyotshna, Bawankule DU, Shanker K, Ahmad A, Tandon S. Standardization and xanthine oxidase inhibitory potential of Zanthoxylum armatum fruits. J Ethnopharmacol. 2019;230:1-8.

\section{Publisher's Note}

Springer Nature remains neutral with regard to jurisdictional claims in published maps and institutional affiliations.
Ready to submit your research? Choose BMC and benefit from:

- fast, convenient online submission

- thorough peer review by experienced researchers in your field

- rapid publication on acceptance

- support for research data, including large and complex data types

- gold Open Access which fosters wider collaboration and increased citations

- maximum visibility for your research: over $100 \mathrm{M}$ website views per year

At BMC, research is always in progress.

Learn more biomedcentral.com/submissions 